\title{
A STUDY ON A RELATION BETWEEN TWO SUMMABILITY METHODS
}

\author{
W. T. SULAIMAN
}

(Communicated by R. Daniel Mauldin)

\begin{abstract}
ABstraCr. Bor $(1985,1986)$ gave a relation between the two summability methods $|C, 1|_{k}$ and $\left|\bar{N}, p_{n}\right|_{k}$ of a series $\sum a_{n}$. These two methods are known to be independent. Generalizing the case, here we introduce relations between the two summability methods $|C, \alpha|_{k}$ and $\left|\bar{N}, p_{n}\right|_{k}$ using multipliers sequence $\left\{\varepsilon_{n}\right\}$.
\end{abstract}

\section{INTRODUCTION}

Let $\sum a_{n}$ be an infinite series of partial sums $s_{n}$. Let $\sigma_{n}^{\delta}$ and $\eta_{n}^{\delta}$ denote the $n$th Cesàro mean of order $\delta(\delta>-1)$ of the sequences $\left\{s_{n}\right\}$ and $\left\{n a_{n}\right\}$ respectively. The series $\sum a_{n}$ is said to be absolutely summable $(C, \delta)$ with index $k$, or simply summable $|C, \delta|_{k}, k \geq 1$, if

$$
\sum_{n=1}^{\infty} n^{k-1}\left|\sigma_{n}^{\delta}-\sigma_{n-1}^{\delta}\right|^{k}<\infty
$$

or equivalently,

$$
\sum_{n=1}^{\infty} n^{-1}\left|\eta_{n}^{\delta}\right|^{k}<\infty
$$

Let $\left\{p_{n}\right\}$ be a sequence of positive real constants such that

$$
P_{n}=\sum_{v=0}^{n} p_{v} \rightarrow \infty \quad \text { as } n \rightarrow \infty\left(P_{-1}=p_{-1}=0\right) .
$$

A series $\sum a_{n}$ is said to be summable $\left|\bar{N}, p_{n}\right|_{k}, k \geq 1$, if

$$
\sum_{n=1}^{\infty}\left(\frac{P_{n}}{p_{n}}\right)^{k-1}\left|T_{n}-T_{n-1}\right|^{k}<\infty \quad \text { (Bor [1]), }
$$

where

$$
T_{n}=P_{n}^{-1} \sum_{v=0}^{n} p_{v} s_{v}
$$

Received by the editors May 15, 1989 and, in revised forms, September 20, 1989, December 21, 1989, and March 27, 1990.

1991 Mathematics Subject Classification. Primary 40G99. 
If we take $p_{n}=1$, then $\left|\bar{N}, p_{n}\right|_{k}$ summability is equivalent to $|C, 1|_{k}$ summability. $\left|\bar{N}, p_{n}\right|_{1}$ is the same as $\left|\bar{N}, p_{n}\right|$. In general the two methods $|C, \delta|_{k}$ and $\left|\bar{N}, p_{n}\right|_{k}$ are not comparable.

Bor established the following two results:

Theorem A. Let $\left\{p_{n}\right\}$ be a sequence of positive real constants such that as $n \rightarrow$ $\infty$

$$
\left\{\begin{array}{l}
\text { (i) } n p_{n}=O\left(P_{n}\right), \\
\text { (ii) } P_{n}=O\left(n p_{n}\right) .
\end{array}\right.
$$

If $\sum a_{n}$ is summable $|C, 1|_{k}$, then it is summable $\left|\bar{N}, p_{n}\right|_{k}, k \geq 1$.

Theorem B. Let $\left\{p_{n}\right\}$ be a sequence of positive real constants such that it satisfies (I). If $\sum a_{n}$ is summable $\left|\bar{N}, p_{n}\right|_{k}$, then it is summable $|C, 1|_{k}, k \geq 1$.

\section{MAIN Results}

We prove the following:

Theorem 1. (A) Let $\left\{p_{n}\right\}$ be a sequence of positive numbers. Let $T_{n}$ be the $\left(\bar{N}, p_{n}\right)$-mean of the series $\sum a_{n}$. If

$$
\begin{aligned}
& \sum_{n=1}^{\infty} n^{k-1}\left|\varepsilon_{n}\right|^{k}\left|\Delta T_{n-1}\right|^{k}<\infty, \\
& \sum_{n=1}^{\infty} n^{k-k \alpha-1}\left(\frac{P_{n}}{p_{n}}\right)^{k}\left|\varepsilon_{n}\right|^{k}\left|\Delta T_{n-1}\right|^{k}<\infty, \quad(0<\alpha<1) \\
& \sum_{n=1}^{\infty} n^{-1}\left(\frac{P_{n}}{p_{n}}\right)^{k}\left|\varepsilon_{n}\right|^{k}\left|\Delta T_{n-1}\right|^{k}<\infty, \quad(\alpha \geq 1) \\
& \sum_{n=1}^{\infty} n^{k-1}\left(\frac{P_{n}}{p_{n}}\right)^{k}\left|\Delta \varepsilon_{n}\right|^{k}\left|\Delta T_{n-1}\right|^{k}<\infty,
\end{aligned}
$$

then the series $\sum a_{n} \varepsilon_{n}$ is summable $|C, \alpha|_{k}, k \geq 1, \alpha>0$.

(B) Let $\left\{p_{n}\right\}$ be a sequence of positive numbers such that (I) holds. Let $\left\{\lambda_{n}\right\},\left\{\varepsilon_{n}\right\}$ be such that $\left\{\lambda_{n}\right\}$ is nonnegative, nondecreasing, $n^{1-\alpha} \lambda_{n}\left|\varepsilon_{n}\right|=O(1)$ for $0<\alpha<1, \lambda_{n}\left|\varepsilon_{n}\right|=O(1)$ and $\varepsilon_{n}=o(1)$ for $\alpha \geq 1, \Delta \varepsilon_{n}=O\left(n^{-1}\left|\varepsilon_{n}\right|\right)$, and

$$
\sum_{n=1}^{m}\left(\frac{P_{n}}{p_{n}}\right)^{k-1}\left|\Delta T_{n-1}\right|^{k}=O\left(\lambda_{m}^{k}\right), \quad m \rightarrow \infty
$$

then in order to have the series $\sum a_{n} \varepsilon_{n}$ summable $|C, \alpha|_{k}$, it is sufficient that

$$
\sum_{n=1}^{\infty} n^{2-\alpha} \lambda_{n}\left|\Delta^{2} \varepsilon_{n}\right|<\infty, \quad(0<\alpha<1)
$$

and

$$
\sum_{n=1}^{\infty} n \lambda_{n}\left|\Delta^{2} \varepsilon_{n}\right|<\infty, \quad(\alpha \geq 1) .
$$


Theorem 2. (A) Let $\left\{p_{n}\right\}$ be a sequence of positive numbers. Let $t_{n}^{1}$ be the nth $(C, 1)$-mean of the sequence $\left\{n a_{n}\right\}$. If

$$
\begin{gathered}
\sum_{n=1}^{\infty} \frac{p_{n}}{P_{n}}\left|\varepsilon_{n}\right|^{k}\left|t_{n}^{1}\right|^{k}<\infty, \\
\sum_{n=1}^{\infty} \frac{1}{n^{k}}\left(\frac{P_{n}}{p_{n}}\right)^{k-1}\left|\varepsilon_{n}\right|^{k}\left|t_{n}^{1}\right|^{k}<\infty, \\
\sum_{n=1}^{\infty}\left(\frac{P_{n}}{p_{n}}\right)^{k-1}\left|\Delta \varepsilon_{n}\right|^{k}\left|t_{n}^{1}\right|^{k}<\infty,
\end{gathered}
$$

then the series $\sum a_{n} \varepsilon_{n}$ is summable $\left|\bar{N}, p_{n}\right|_{k}, k \geq 1$.

(B) Let $\left\{p_{n}\right\}$ be a sequence of positive numbers such that (I) holds. Let $\left\{\lambda_{n}\right\},\left\{\varepsilon_{n}\right\}$ be such that $\left\{\lambda_{n}\right\}$ is nonnegative, nondecreasing, $\lambda_{n}\left|\varepsilon_{n}\right|=O(1)$, $\varepsilon_{n}=o(1), \Delta \varepsilon_{n}=O\left(n^{-1}\left|\varepsilon_{n}\right|\right)$, and

$$
\sum_{n=1}^{m} n^{-1}\left|t_{n}^{1}\right|^{k}=O\left(\lambda_{m}^{k}\right)
$$

then in order to have the series $\sum a_{n} \varepsilon_{n}$ summable $\left|\bar{N}, p_{n}\right|_{k}, k \geq 1$, it is sufficient that

$$
\sum_{n=1}^{\infty} n \lambda_{n}\left|\Delta^{2} \varepsilon_{n}\right|<\infty
$$

\section{ReQuired LeMMA}

Lemma. If $\sigma>\delta>0$, then

$$
\sum_{n=v+1}^{m} \frac{(n-v)^{\delta-1}}{n^{\sigma}}=O\left(v^{\delta-\sigma}\right), \quad \text { as } m \rightarrow \infty
$$

\section{Proof of THE THEOREMS}

Proof of Theorem 1. (A) Let $t_{n}^{\alpha}$ be the $n$th $(C, \alpha)$-mean, $\alpha>0$, of the sequence $\left\{n a_{n} \varepsilon_{n}\right\}$. Then we have

$$
t_{n}^{\alpha}=\frac{1}{A_{n}^{\alpha}} \sum_{v=1}^{n} A_{n-v}^{\alpha-1} v a_{v} \varepsilon_{v}
$$

where

$$
A_{n}^{\delta}=\left(\begin{array}{c}
n+\delta \\
n
\end{array}\right)=\frac{(\delta+1)(\delta+2) \cdots(\delta+n)}{n !} \simeq \frac{n^{\delta}}{\Gamma(\delta+1)}, \quad \delta \neq-1,-2, \ldots
$$

As

$$
T_{n}=\frac{1}{P_{n}} \sum_{v=0}^{n} p_{v} \sum_{r=0}^{v} a_{r}=\frac{1}{P_{n}} \sum_{v=0}^{n}\left(P_{n}-P_{v-1}\right) a_{v}
$$

then

$$
T_{n}-T_{n-1}=\frac{p_{n}}{P_{n} P_{n-1}} \sum_{v=1}^{n} P_{v-1} a_{v}
$$




$$
\begin{aligned}
& t_{n}^{\alpha}= \frac{1}{A_{n}^{\alpha}} \sum_{v=1}^{n} P_{v-1} a_{v}\left\{v A_{n-v}^{\alpha-1} P_{v-1}^{-1} \varepsilon_{v}\right\} \\
&=\frac{1}{A_{n}^{\alpha}}\left[\sum_{v=1}^{n-1}\left\{\sum_{r=1}^{v} P_{r-1} a_{r}\right\} \Delta\left\{v A_{n-v}^{\alpha-1} P_{v-1}^{-1} \varepsilon_{v}\right\}+\left\{\sum_{r=1}^{n} P_{r-1} a_{r}\right\} n P_{n-1}^{-1} \varepsilon_{n}\right] \\
&=-\frac{1}{A_{n}^{\alpha}}\left[\sum _ { v = 1 } ^ { n - 1 } \left\{v A_{n-v}^{\alpha-1} \varepsilon_{v} \Delta T_{v-1}+\frac{P_{v-1}}{p_{v}} A_{n-v}^{\alpha-1} \varepsilon_{v} \Delta T_{v-1}+(v+1) \frac{P_{v-1}}{p_{v}}\right.\right. \\
& \quad \times \Delta A_{n-v}^{\alpha-1} \varepsilon_{v} \Delta T_{v-1} \\
&\left.\left.\quad+(v+1) \frac{P_{v-1}}{p_{v}} A_{n-v-1}^{\alpha-1} \Delta \varepsilon_{v} \Delta T_{v-1}\right\}+n \frac{P_{n}}{p_{n}} \varepsilon_{n} \Delta T_{n-1}\right] \\
&=t_{n, 1}^{\alpha}+t_{n, 2}^{\alpha}+t_{n, 3}^{\alpha}+t_{n, 4}^{\alpha}+t_{n, 5}^{\alpha} .
\end{aligned}
$$

In order to prove the theorem, by Minkowski's inequality, it is sufficient to show that

$$
\sum_{n=1}^{\infty} n^{-1}\left|t_{n, r}^{\alpha}\right|^{k}<\infty, \quad r=1,2,3,4,5
$$

Applying Hölder's inequality, we have

$$
\begin{aligned}
\sum_{n=2}^{m+1} n^{-1}\left|t_{n, 1}^{\alpha}\right|^{k} & \leq \sum_{n=2}^{m+1} \frac{1}{n A_{n}^{\alpha}} \sum_{v=1}^{n-1} v^{k} A_{n-v}^{\alpha-1}\left|\varepsilon_{v}\right|^{k}\left|\Delta T_{v-1}\right|^{k}\left\{\sum_{v=1}^{n-1} \frac{A_{n-v}^{\alpha-1}}{A_{n}^{\alpha}}\right\} \\
& =O(1) \sum_{v=1}^{m} v^{k}\left|\varepsilon_{v}\right|^{k}\left|\Delta T_{v-1}\right|^{k} \sum_{n=v+1}^{m+1} \frac{A_{n-v}^{\alpha-1}}{n A_{n}^{\alpha}} \\
& =O(1) \sum_{v=1}^{m} v^{k}\left|\varepsilon_{v}\right|^{k}\left|\Delta T_{v-1}\right|^{k} \sum_{n=v+1}^{m+1} \frac{(n-v)^{\alpha-1}}{n^{1+\alpha}} \\
& =O(1) \sum_{v=1}^{m} v^{k-1}\left|\varepsilon_{v}\right|^{k}\left|\Delta T_{v-1}\right|^{k} \cdot \\
\sum_{n=2}^{m+1} n^{-1}\left|t_{n, 2}^{\alpha}\right|^{k} \leq & \sum_{n=2}^{m+1} \frac{1}{n A_{n}^{\alpha}} \sum_{v=1}^{n-1}\left(\frac{P_{v}}{p_{v}}\right)^{k} A_{n-v}^{\alpha-1}\left|\varepsilon_{v}\right|^{k}\left|\Delta T_{v-1}\right|^{k}\left\{\sum_{v=1}^{n-1} \frac{A_{n-v}^{\alpha-1}}{A_{n}^{\alpha}}\right\} \\
= & O(1) \sum_{v=1}^{m}\left(\frac{P_{v}}{p_{v}}\right)^{k}\left|\varepsilon_{v}\right|^{k}\left|\Delta T_{v-1}\right|^{k} \sum_{n=v+1}^{m+1} \frac{A_{n-v}^{\alpha-1}}{n A_{n}^{\alpha}} \\
= & O(1) \sum_{v=1}^{m} v^{-1}\left(\frac{P_{v}}{p_{v}}\right)^{k}\left|\varepsilon_{v}\right|^{k}\left|\Delta T_{v-1}\right|^{k}
\end{aligned}
$$




$$
\begin{aligned}
& \sum_{n=2}^{m+1} n^{-1}\left|t_{n, 4}^{\alpha}\right|^{k} \leq \sum_{n=2}^{m+1} \frac{1}{n A_{n}^{\alpha}} \sum_{v=1}^{n-1}(v+1)^{k}\left(\frac{P_{v}}{p_{v}}\right)^{k} A_{n-v-1}^{\alpha-1}\left|\Delta \varepsilon_{v}\right|^{k}\left|\Delta T_{v-1}\right|^{k} \\
& \times\left\{\sum_{v=1}^{n-1} \frac{A_{n-v-1}^{\alpha-1}}{A_{n}^{\alpha}}\right\}^{k-1} \\
&= O(1) \sum_{v=1}^{m} v^{k}\left(\frac{P_{v}}{p_{v}}\right)^{k}\left|\Delta \varepsilon_{v}\right|^{k}\left|\Delta T_{v-1}\right|^{k} \\
& \times \sum_{n=v+1}^{m+1} \frac{A_{n-v-1}^{\alpha-1}}{n A_{n}^{\alpha}} \\
&= O(1) \sum_{v=1}^{m} v^{k-1}\left(\frac{P_{v}}{p_{v}}\right)^{k}\left|\Delta \varepsilon_{v}\right|^{k}\left|\Delta T_{v-1}\right|^{k} . \\
& \sum_{n=1}^{m} n^{-1}\left|t_{n, 5}^{\alpha}\right|^{k}=O(1) \sum_{n=1}^{m} n^{k-k \alpha-1}\left(\frac{P_{n}}{p_{n}}\right)^{k}\left|\varepsilon_{n}\right|^{k}\left|\Delta T_{n-1}\right|^{k}
\end{aligned}
$$

For $t_{n, 3}^{\alpha}$ the two cases $0<\alpha<1$, and $\alpha \geq 1$ need to be considered. For $0<\alpha<1$, we have

$$
\begin{aligned}
\sum_{n=2}^{m+1} n^{-1}\left|t_{n, 3}^{\alpha}\right|^{k} \leq & \sum_{n=2}^{m+1} \frac{1}{n\left(A_{n}^{\alpha}\right)^{k}} \sum_{v=1}^{n-1}(v+1)^{k}\left(\frac{P_{v}}{p_{v}}\right)^{k}\left|\Delta A_{n-v}^{\alpha-1}\right|\left|\varepsilon_{v}\right|^{k}\left|\Delta T_{v-1}\right|^{k} \\
& \times\left\{\sum_{v=1}^{n-1}\left|\Delta A_{n-v}^{\alpha-1}\right|\right\} \\
= & O(1) \sum_{n=2}^{m+1} \frac{1}{n^{1+k \alpha}} \sum_{v=1}^{n-1} v^{k}\left(\frac{P_{v}}{p_{v}}\right)^{k}(n-v)^{\alpha-2}\left|\varepsilon_{v}\right|^{k}\left|\Delta T_{v-1}\right|^{k} \\
& \times\left\{\sum_{v=1}^{n-1}(n-v)^{\alpha-2}\right\}^{k-1} \\
= & O(1) \sum_{v=1}^{m} v^{k}\left(\frac{P_{v}}{p_{v}}\right)^{k}\left|\varepsilon_{v}\right|^{k}\left|\Delta T_{v-1}\right|^{k} \\
& \times \sum_{n=v+1}^{m+1} \frac{(n-v)^{\alpha-2}}{n^{1+k \alpha}} \\
= & O(1) \sum_{v=1}^{m} v^{k-k \alpha-1}\left(\frac{P_{v}}{p_{v}}\right)^{k}\left|\varepsilon_{v}\right|^{k}\left|\Delta T_{v-1}\right|^{k}
\end{aligned}
$$

When $\alpha=1, \Delta A_{n-v}^{\alpha-1}=0$, hence $t_{n, 3}^{\alpha}=0$. It remains to consider $t_{n, 3}^{\alpha}$ for 
$\alpha>1$, and for this case we have

$$
\begin{aligned}
\sum_{n=2}^{m+1} n^{-1}\left|t_{n, 3}^{\alpha}\right|^{k}= & O(1) \sum_{n=2}^{m+1} \frac{1}{n^{1+k \alpha}} \sum_{v=1}^{n-1} v^{k}\left(\frac{P_{v}}{p_{v}}\right)^{k}(n-v)^{\alpha-2} \frac{\left|\varepsilon_{v}\right|^{k}\left|\Delta T_{v-1}\right|^{k}}{\cdot n^{(\alpha-1)(k-1)}} \\
\left(\text { as } \sum_{v=1}^{n-1}(n-v)^{\alpha-2}=\right. & \left.O(1) \int_{1}^{n-1}(n-x)^{\alpha-2} d x=O\left(n^{\alpha-1}\right) .\right) \\
= & O(1) \sum_{v=1}^{m} v^{k}\left(\frac{P_{v}}{p_{v}}\right)^{k}\left|\varepsilon_{v}\right|^{k}\left|\Delta T_{v-1}\right|^{k} \\
& \times \sum_{n=v+1}^{m+1} \frac{(n-v)^{\alpha-2}}{n^{k+\alpha}} \\
= & O(1) \sum_{v=1}^{m} v^{-1}\left(\frac{P_{v}}{p_{v}}\right)^{k}\left|\varepsilon_{v}\right|^{k}\left|\Delta T_{v-1}\right|^{k} .
\end{aligned}
$$

This completes the proof of (A). To prove (B), by (A), it is sufficient to show that the conditions $(2.1)-(2.4)$ are all satisfied. Since $\Delta \varepsilon_{n}=O\left(n^{-1}\left|\varepsilon_{n}\right|\right)$, then by (I) each of the summations in (2.1) and (2.4) is equal to

$$
\begin{aligned}
O(1) & \sum_{n=1}^{\infty} n^{-1}\left(\frac{P_{n}}{p_{n}}\right)^{k}\left|\varepsilon_{n}\right|^{k}\left|\Delta T_{n-1}\right|^{k} \\
& = \begin{cases}O(1) \sum_{n=1}^{\infty} n^{k-k \alpha-1}\left(\frac{P_{n}}{p_{n}}\right)^{k}\left|\varepsilon_{n}\right|^{k}\left|\Delta T_{n-1}\right|^{k} & (0<\alpha<1), \\
O(1) \sum_{n=1}^{\infty} n^{-1}\left(\frac{P_{n}}{p_{n}}\right)^{k}\left|\varepsilon_{n}\right|^{k}\left|\Delta T_{n-1}\right|^{k}, & (\alpha \geq 1) .\end{cases}
\end{aligned}
$$

Therefore, only the two conditions (2.2) and (2.3) are left to be considered. For $0<\alpha<1$, we have

$$
\begin{aligned}
\sum_{v=1}^{m} v^{k-k \alpha-1}\left(\frac{P_{v}}{p_{v}}\right)^{k}\left|\varepsilon_{v}\right|^{k}\left|\Delta T_{v-1}\right|^{k} \\
=O(1) \sum_{v=1}^{m} v^{k-k \alpha}\left|\varepsilon_{v}\right|^{k}\left(\frac{P_{v}}{p_{v}}\right)^{k-1}\left|\Delta T_{v-1}\right|^{k} \\
=O(1) \sum_{v=1}^{m-1} \sum_{r=1}^{v}\left(\frac{P_{r}}{p_{r}}\right)^{k-1}\left|\Delta T_{r-1}\right|^{k}\left\{v^{k-k \alpha-1}\left|\varepsilon_{v}\right|^{k}+(v+1)^{k-k \alpha} \Delta\left|\varepsilon_{v}\right|^{k}\right\} \\
\quad+O(1) \sum_{r=1}^{m}\left(\frac{P_{r}}{p_{r}}\right)^{k-1}\left|\Delta T_{r-1}\right|^{k} \cdot m^{k-k \alpha}\left|\varepsilon_{m}\right|^{k} \\
=I_{1}+I_{2}+I_{3}, \quad \text { say. }
\end{aligned}
$$




$$
\begin{aligned}
I_{1} & =O(1) \sum_{v=1}^{\infty} v^{k-k \alpha-1} \lambda_{v}^{k}\left|\varepsilon_{v}\right|^{k}=O(1) \sum_{v=1}^{\infty} v^{-\alpha} \lambda_{v}\left|\varepsilon_{v}\right| \\
& =O(1) \sum_{v=1}^{\infty} v^{-\alpha} \lambda_{v}\left|\sum_{r=v}^{\infty} \Delta \varepsilon_{r}\right|, \quad \text { as } \varepsilon_{m}=O(1)=O(1) \sum_{v=1}^{\infty} v^{-\alpha} \lambda_{v} \sum_{r=v}^{\infty}\left|\Delta \varepsilon_{r}\right| \\
& =O(1) \sum_{r=1}^{\infty}\left|\Delta \varepsilon_{r}\right| \sum_{v=1}^{r} v^{-\alpha} \lambda_{v}=O(1) \sum_{r=1}^{\infty}\left|\Delta \varepsilon_{r}\right| \lambda_{r} \int_{1}^{r} x^{-\alpha} d x \\
& =O(1) \sum_{r=1}^{\infty} r^{1-\alpha} \lambda_{r}\left|\Delta \varepsilon_{r}\right|=O(1) \sum_{r=1}^{\infty} r^{1-\alpha} \lambda_{r} \sum_{v=r}^{\infty}\left|\Delta^{2} \varepsilon_{r}\right|, \quad \text { as } \Delta \varepsilon_{m}=o(1) \\
& =O(1) \sum_{v=1}^{\infty}\left|\Delta^{2} \varepsilon_{v}\right| \sum_{r=1}^{v} r^{1-\alpha} \lambda_{r} \\
& =O(1) \sum_{v=1}^{\infty} v^{2-\alpha} \lambda_{v}\left|\Delta^{2} \varepsilon_{v}\right| .
\end{aligned}
$$

Since

$$
\Delta\left|\varepsilon_{v}\right|^{k}=K\left(\left|\varepsilon_{v}\right|-\left|\varepsilon_{v+1}\right|\right) \xi^{k-1},
$$

for some $\xi$ between $\left|\varepsilon_{v}\right|$ and $\left|\varepsilon_{v+1}\right|$, by the mean value theorem,

$$
\begin{aligned}
\Delta\left|\varepsilon_{v}\right|^{k} & =O\left\{\left|\varepsilon_{v}\right|^{k-1} \Delta\left|\varepsilon_{v}\right|\right\}, \quad \text { as } \varepsilon_{m}=o(1) \\
& =O\left\{\left|\varepsilon_{v}\right|^{k-1}\left|\Delta \varepsilon_{v}\right|\right\},
\end{aligned}
$$

therefore,

$$
\begin{aligned}
I_{2} & =O(1) \sum_{v=1}^{\infty} v^{k-k \alpha} \lambda_{v}^{k} \Delta\left|\varepsilon_{v}\right|^{k} \\
& =O(1) \sum_{v=1}^{\infty} v^{k-k \alpha} \lambda_{v}^{k}\left|\varepsilon_{v}\right|^{k-1}\left|\Delta \varepsilon_{v}\right| \\
& =O(1) \sum_{v=1}^{\infty} v^{1-\alpha} \lambda_{v}\left|\Delta \varepsilon_{v}\right|=O(1) \sum_{v=1}^{\infty} v^{1-\alpha} \lambda_{v} \sum_{r=v}^{\infty}\left|\Delta^{2} \varepsilon_{r}\right| \\
& =O(1) \sum_{r=1}^{\infty} r^{2-\alpha} \lambda_{r}\left|\Delta^{2} \varepsilon_{r}\right| \\
I_{3} & =O(1) m^{k-k \alpha} \lambda_{m}^{k}\left|\varepsilon_{m}\right|^{k}=O(1) .
\end{aligned}
$$

Now, for the case $\alpha \geq 1$ we have

$$
\begin{aligned}
\sum_{v=1}^{m} v^{-1}\left(\frac{P_{v}}{p_{v}}\right)^{k}\left|\varepsilon_{v}\right|^{k}\left|\Delta T_{v-1}\right|^{k} & \\
= & O(1) \sum_{v=1}^{m}\left|\varepsilon_{v}\right|^{k}\left(\frac{P_{v}}{p_{v}}\right)^{k-1}\left|\Delta T_{v-1}\right|^{k} \\
& =O(1) \sum_{v=1}^{m-1} \sum_{r=1}^{v}\left(\frac{P_{r}}{p_{r}}\right)^{k-1}\left|\Delta T_{r-1}\right|^{k} \Delta\left|\varepsilon_{v}\right|^{k}+O(1) \sum_{r=1}^{m}\left(\frac{P_{r}}{p_{r}}\right)^{k-1}\left|\Delta T_{r-1}\right|^{k}\left|\varepsilon_{m}\right|^{k} \\
& =J_{1}+J_{2}, \quad \text { say. }
\end{aligned}
$$




$$
\begin{aligned}
J_{1} & =O(1) \sum_{v=1}^{\infty} \lambda_{v}^{k}\left|\varepsilon_{v}\right|^{k-1}\left|\Delta \varepsilon_{v}\right|=O(1) \sum_{v=1}^{\infty} \lambda_{v}\left|\Delta \varepsilon_{v}\right| \\
& =O(1) \sum_{v=1}^{\infty} v \lambda_{v}\left|\Delta^{2} \varepsilon_{v}\right|{ }_{v} \lambda_{v}\left|\Delta^{2} \varepsilon_{v}\right|, \quad \text { as before. } \\
J_{2} & =O(1) \lambda_{m}^{k}\left|\varepsilon_{m}\right|^{k}=O(1) .
\end{aligned}
$$

This completes the proof of Theorem 1.

Proof of Theorem 2. (A) Let $Q_{n}$ denote the $\left(\bar{N}, p_{n}\right)$-mean of the series $\sum a_{n} \varepsilon_{n}$. Then we have

$$
\begin{aligned}
& Q_{n}= \frac{1}{P_{n}} \sum_{v=0}^{n} p_{v} \sum_{r=0}^{v} a_{r} \varepsilon_{r}=\frac{1}{P_{n}} \sum_{v=0}^{n}\left(P_{n}-P_{v-1}\right) a_{v} \varepsilon_{v} \\
& Q_{n}-Q_{n-1}=\frac{p_{n}}{P_{n} P_{n-1}} \sum_{v=1}^{n} P_{v-1} a_{v} \varepsilon_{v} . \\
& Q_{n}-Q_{n-1}=\frac{p_{n}}{P_{n} P_{n-1}} \sum_{v=1}^{n} v a_{v} \frac{P_{v-1} \varepsilon_{v}}{v} \\
&=\frac{p_{n}}{P_{n} P_{n-1}}\left[\sum_{v=1}^{n-1}(v+1) t_{v}^{1}\left\{-\frac{p_{v} \varepsilon_{v}}{v}+\frac{P_{v} \varepsilon_{v}}{v(v+1)}+\frac{P_{v} \Delta \varepsilon_{v}}{v+1}\right\}\right. \\
&\left.=Q_{n, 1}+Q_{n, 2}+Q_{n, 3}+Q_{n, 4} .+\frac{n+1}{n} P_{n-1} \varepsilon_{n} t_{n}^{1}\right]
\end{aligned}
$$

In order to prove the theorem it is sufficient, by Minkowski's inequality, to show that

$$
\sum_{n=1}^{\infty}\left(\frac{P_{n}}{p_{n}}\right)^{k-1}\left|Q_{n, r}\right|^{k}<\infty, \quad r=1,2,3,4
$$

Applying Hölder's inequality, we have

$$
\begin{aligned}
\sum_{n=2}^{m+1}\left(\frac{P_{n}}{p_{n}}\right)^{k-1}\left|Q_{n, 1}\right|^{k} & \leq \sum_{n=2}^{m+1} \frac{p_{n}}{P_{n} P_{n-1}} \sum_{v=1}^{n-1}\left(1+\frac{1}{v}\right)^{k} p_{v}\left|\varepsilon_{v}\right|^{k}\left|t_{v}^{1}\right|^{k}\left\{\sum_{v=1}^{n-1} \frac{p_{v}}{P_{n-1}}\right\}^{k-1} \\
& =O(1) \sum_{v=1}^{m} p_{v}\left|\varepsilon_{v}\right|^{k}\left|t_{v}^{1}\right|^{k} \sum_{n=v+1}^{m+1} \frac{p_{n}}{P_{n} P_{n-1}} \\
& =O(1) \sum_{v=1}^{m} \frac{p_{v}}{P_{v}}\left|\varepsilon_{v}\right|^{k}\left|t_{v}^{1}\right|^{k}
\end{aligned}
$$




$$
\begin{aligned}
\sum_{n=2}^{m+1}\left(\frac{P_{n}}{p_{n}}\right)^{k-1}\left|Q_{n, 2}\right|^{k} & \leq \sum_{n=2}^{m+1} \frac{p_{n}}{P_{n} P_{n-1}} \sum_{v=1}^{n-1} \frac{1}{v^{k}}\left(\frac{P_{v}}{p_{v}}\right)^{k} p_{v}\left|\varepsilon_{v}\right|^{k}\left|t_{v}^{1}\right|^{k}\left\{\sum_{v=1}^{n-1} \frac{p_{v}}{P_{n-1}}\right\}^{k-1} \\
& =O(1) \sum_{v=1}^{m} \frac{1}{v^{k}}\left(\frac{P_{v}}{p_{v}}\right)^{k} p_{v}\left|\varepsilon_{v}\right|^{k}\left|t_{v}^{1}\right|^{k} \sum_{n=v+1}^{m+1} \frac{p_{n}}{P_{n} P_{n-1}} \\
& =O(1) \sum_{v=1}^{m} \frac{1}{v^{k}}\left(\frac{P_{v}}{p_{v}}\right)^{k-1}\left|\varepsilon_{v}\right|^{k}\left|t_{v}^{1}\right|^{k} \\
\sum_{n=2}^{m+1}\left(\frac{P_{n}}{p_{n}}\right)^{k-1}\left|Q_{n, 3}\right|^{k} & \leq \sum_{n=2}^{m+1} \frac{p_{n}}{P_{n} P_{n-1}} \sum_{v=1}^{n-1}\left(\frac{P_{v}}{p_{v}}\right)^{k} p_{v}\left|\Delta \varepsilon_{v}\right|^{k}\left|t_{v}^{1}\right|^{k}\left\{\sum_{v=1}^{n-1} \frac{p_{v}}{P_{n-1}}\right\}^{k-1}
\end{aligned}
$$$$
=O(1) \sum_{v=1}^{m}\left(\frac{P_{v}}{p_{v}}\right)^{k} p_{v}\left|\Delta \varepsilon_{v}\right|^{k}\left|t_{v}^{1}\right|^{k} \sum_{n=v+1}^{m+1} \frac{p_{n}}{P_{n} P_{n-1}}
$$$$
=O(1) \sum_{v=1}^{m}\left(\frac{P_{v}}{p_{v}}\right)^{k-1}\left|\Delta \varepsilon_{v}\right|^{k}\left|t_{v}^{1}\right|^{k} \text {. }
$$

$\sum_{n=1}^{m}\left(\frac{P_{n}}{p_{n}}\right)^{k-1}\left|Q_{n, 4}\right|^{k}=O(1) \sum_{n=1}^{m} \frac{p_{n}}{P_{n}}\left|\varepsilon_{n}\right|^{k}\left|t_{n}^{1}\right|^{k}$.

The proof of (B) can be achieved exactly as in the case of Theorem 1. This completes the proof of Theorem 2 .

\section{APPLICATIONS AND COROLLARIES}

1. If we take $\alpha=1$ and $\varepsilon_{n}=1$ for all $n$ in Theorem 1 (A), we get Theorem B provided (I) holds.

2. If we take $\alpha=1$ and $\varepsilon_{n}=1$ for all $n$ in Theorem 2 (A), we get Theorem A provided (I) holds.

Corollary 1. Let $\left\{p_{n}\right\}$ be a sequence of positive real constants such that $n p_{n}=$ $O\left(P_{n}\right)$. Then sufficient conditions that $\sum a_{n} \varepsilon_{n}$ be summable $|C, \alpha|_{k}, k \geq 1$, $\alpha>0$, whenever $\sum a_{n}$ is summable $\left|\bar{N}, p_{n}\right|_{k}$ are

(i) $\left|\varepsilon_{n}\right|=O\left\{n^{\alpha-1+1 / k}\left(p_{n} / P_{n}\right)^{1 / k}\right\} \quad(\alpha<1)$,

(ii) $\left|\varepsilon_{n}\right|=O\left\{\left(n p_{n} / P_{n}\right)^{1 / k}\right\} \quad(\alpha \geq 1)$,

(iii) $\left|\Delta \varepsilon_{n}\right|=O\left\{n^{-1+1 / k}\left(p_{n} / P_{n}\right)^{1 / k}\right\}$.

Proof. Since $n p_{n}=O\left(P_{n}\right)$, then, for $0<\alpha<1$,

$$
\begin{aligned}
\sum_{n=1}^{\infty} n^{k-1}\left|\varepsilon_{n}\right|^{k}\left|\Delta T_{n-1}\right|^{k} & =O(1) \sum_{n=1}^{\infty} n^{k \alpha}\left(\frac{p_{n}}{P_{n}}\right)^{k}\left(\frac{P_{n}}{p_{n}}\right)^{k-1}\left|\Delta T_{n-1}\right|^{k} \\
& =O(1) \sum_{n=1}^{\infty} n^{k \alpha-k}\left(\frac{P_{n}}{p_{n}}\right)^{k-1}\left|\Delta T_{n-1}\right|^{k} \\
& =O(1) \sum_{n=1}^{\infty}\left(\frac{P_{n}}{p_{n}}\right)^{k-1}\left|\Delta T_{n-1}\right|^{k}=O(1) .
\end{aligned}
$$

Similarly, we can show that (2.2)-(2.4) are also satisfied, and the result follows by Theorem 1 (A). 
Corollary 2. Let $\left\{p_{n}\right\}$ be a sequence of positive real constants such that $n p_{n}=$ $O\left(P_{n}\right)$. Then sufficient conditions that $\sum a_{n} \varepsilon_{n}$ be summable $\left|\bar{N}, p_{n}\right|_{k}, k \geq 1$, whenever $\sum a_{n}$ is summable $|C, 1|_{k}$ are

(i) $\left|\varepsilon_{n}\right|=O\left\{\left(n p_{n} / P_{n}\right)^{1-1 / k}\right\}$,

(ii) $\left|\Delta \varepsilon_{n}\right|=O\left\{n^{-1 / k}\left(p_{n} / P_{n}\right)^{1-1 / k}\right\}$.

Proof. Since $n p_{n}=O\left(P_{n}\right)$, then $\left|\varepsilon_{n}\right|=O(1)$. Hence

$$
\sum_{n=1}^{\infty} \frac{p_{n}}{P_{n}}\left|\varepsilon_{n}\right|^{k}\left|t_{n}^{1}\right|^{k}=O(1) \sum_{n=1}^{\infty} \frac{1}{n}\left|t_{n}^{1}\right|^{k}=O(1) .
$$

Conditions (2.6) and (2.7) are also satisfied, and the result follows by Theorem 2 (A).

\section{ACKNOWLEDGMENT}

I am so indebted to the referee who guided me for a good adjustment of this paper.

\section{REFERENCES}

1. H. Bor, On two summability methods, Math. Proc. Cambridge Soc. 97 (1985), 147-149.

2. __ A note on two summability methods, Proc. Amer. Math. Soc. 98 (1986), 81-84.

Department of Applied Sciences, College of Technological Studies, P.O. Box 42325, 70654 KUWAIT 